\title{
Role of the CTRP family in tumor development and progression (Review)
}

\author{
MOWEI KONG ${ }^{1}$, YU GAO ${ }^{1}$, XIANG GUO ${ }^{2}$, YUYU XIE ${ }^{3}$ and YAMEI YU ${ }^{3}$ \\ Departments of ${ }^{1}$ Endocrinology, ${ }^{2}$ Respiratory, ${ }^{3}$ Dermatology and ${ }^{4}$ Hematology, \\ Affiliated Hospital of Chengde Medical University, Chengde, Hebei 067000, P.R. China
}

Received February 17, 2021; Accepted May 19, 2021

DOI: $10.3892 / \mathrm{ol} .2021 .12984$

\begin{abstract}
C1q tumor necrosis factor-related proteins (CTRPs), which are members of the adipokine superfamily, have gained significant interest in the recent years. CTRPs are homologs of adiponectin with numerous functions and are closely associated with metabolic diseases, such as abnormal glucose and lipid metabolism and diabetes. Previous studies have demonstrated that CTRPs are highly involved in the regulation of numerous physiological and pathological processes, including glycolipid metabolism, protein kinase pathways, cell proliferation, cell apoptosis and inflammation. CTRPs also play important roles in the development and progression of numerous types of tumor, including liver, colon and lung cancers. This observation can be attributed to the fact that diabetes, obesity and insulin resistance are independent risk factors for tumorigenesis. Numerous CTRPs, including CTRP3, CTRP4, CTRP6 and CTRP8, have been reported to be associated with tumor progression by activating multiple signal pathways. CTRPs could therefore be considered as diagnostic markers and therapeutic targets in some cancers. However, the underlying mechanisms of CTRPs in tumorigenesis remain unknown. The present review aimed to determine the roles and underlying mechanisms of CTRPs in tumorigenesis, which may help the development of novel cancer treatments in the future.
\end{abstract}

\section{Contents}

1. Introduction

2. CTRP family: General characteristics

3. Osteosarcoma (OS) and CTRP3

4. Role of CTRP4 and CTRP6 in liver cancer

5. Roles of CTRP4 and CTRP6 in colon cancer

Correspondence to: Dr Yu Gao, Department of Endocrinology, Affiliated Hospital of Chengde Medical University, 36 Nanyingzi Street, Chengde, Hebei 067000, P.R. China

E-mail:yugao815@163.com

Key words: tumor, Clq tumor necrosis factor-related protein, biomarker, metabolism
6. GBM and CTRP8

7. Tumor-related roles of the other CTRP family members

8. Conclusions

\section{Introduction}

Due to the aging population and numerous environmental factors, the incidence of malignant tumors is increasing, which poses a major threat to human health $(1,2)$. Patients with malignant tumors often experience very subtle signs and symptoms. This, combined with a lack of early diagnostic methods, lead to diagnosis at advanced stages and missed opportunity for early and effective treatments. In addition, due to the heterogeneity of tumor cells with respect to gene expression, metabolic activity, proliferation and metastatic potential, actual treatment methods often fail to achieve satisfactory therapeutic outcomes. Personalized diagnostic methods and therapeutic options are therefore needed to improve the diagnosis and treatment of malignant tumors (3).

Previous studies have reported that fat metabolism is related to the development and progression of cancer $(4,5)$. Fatty acid metabolism plays a key role in maintaining the microenvironment of malignant tumors, and lipid droplet metabolism has been demonstrated to be highly involved in the development and progression of multiple types of tumor (4). Adiponectin, an adipocytokine secreted by fat cells, exhibits some insulin-sensitizing, anti-diabetic, anti-atherosclerosis and anti-inflammatory properties on all cells (4). Furthermore, adiponectin was reported to be strongly associated with the development of several types of tumor, such as prostate cancer and breast tumor, suggesting its potential role as a therapeutic target and marker (6-8). Subsequently, it is hypothesized that the effects of fat metabolism on tumors may be associated with adiponectin and its homologs.

Complement $\mathrm{Cq} 1 /$ tumor necrosis factor-related protein (CTRP), a member of the adipokine superfamily expressed in and excreted by adipose tissue, has a high degree of sequence homology with adiponectin (9). Previous studies have demonstrated that CTRP family members are involved in the regulation of multiple physiological and pathological processes, including glucose and lipid metabolism, inflammation, cartilage formation and development, myocardial protection and vasodilation $(10,11)$. In addition, CTRPs might 
also be involved in the pathogenesis of multiple sclerosis and may be considered as biomarkers or therapeutic targets (12). The role of the CRTP family in cancer has attracted great interest and is being extensively studies. At present, several CTRP family members are considered as molecular mediators that can regulate tumorigenesis and tumor cell invasion and metastasis $(13,14)$. The present review aimed to determine the pathophysiological roles of CTRP family members in the development and progression of different types of tumor. CTRPs may therefore be considered as potential new targets for future research and clinical treatment of cancers.

\section{CTRP family: General characteristics}

Previous studies have demonstrated that adipose tissue serves as the primary energy storage organ of the body and has potent endocrine functions, since it secretes a variety of adipokines. CTRP is an adipokine superfamily of proteins discovered by Wong et al (15), which are structurally similar to adiponectin (15) and exhibit numerous functions, including blood lipid regulation $(16,17)$, insulin sensitization (18) and anti-inflammatory effects (19). Structurally, CTRPs are predominantly homotrimers; however, some CTRPs exist as heterohexamers (20). Apart from CTRP4, all CTRPs consist of an amino-terminal signal peptide, a short variable region, a collagen-like domain and most importantly, a globular carboxy-terminal domain homologous to the complement protein C1q, which is crucial for their biological functions (9,13) (Fig. 1).

Four CTRP family members, CTRP3, CTRP4, CTRP6 and CTRP8, have been reported to be associated with tumor promotion. In humans, CTRP3 mRNA is expressed in multiple tissues and organs, including adipose tissue, cartilage and the kidney, and it is highly abundant in the lung and spleen $(21,22)$. It was reported that CTRP3 is the closest functional homolog of adiponectin, whereas CTRP4 is a classic secreted protein. Unlike other CTRPs, CTRP4 contains two globular C1q domains in series and lacks a collagen-like domain (23). In mice, increased CTRP4 level has been observed in the heart, liver, brain and kidneys (10). In humans, CTRP4 is expressed in the testes, kidneys, fat and brain. In the brain, it is primarily expressed and secreted by neurons (23). A recent study demonstrated that CTRP4 is not only associated with metabolism but is also involved in the regulation of bone metabolism and the promotion of osteoblast differentiation (24). CTRP6 is extensively expressed in the uterus, placenta, skin, lungs and fat in humans. CTRP6 functions as a novel metabolic/immunomodulator by binding to a variety of microorganisms and endogenous ligands (25) and acts as an intermediate between obesity and the inflammation of adipose tissue or insulin resistance (26). The last tumor-associated CTRP, CTRP8, is the least studied protein. This is partly attributed to the fact that CTRP8 is not expressed in mice, which makes its study more difficult. In humans, CTRP8 is expressed in the lungs and testes (27). It is currently known that CTRP3 promotes the proliferation of osteosarcoma cells (28). CTRP4 and CTRP6 are involved in the survival of human liver cancer cells and tumor tissue angiogenesis (14). CTRP8 enhances the invasiveness of glioblastoma (GBM) (29). The role and underlying mechanisms of other CTRP family members in tumors require further investigation.

\section{Osteosarcoma (OS) and CTRP3}

Previous studies have reported that CTRP3 is strongly associated with OS $(29,30)$, which is the most common malignant bone tumor in adolescents, with an incidence of $\sim 0.0004 \%$ worldwide. CTRP3 is a growth factor which presents two subtypes in humans, CTRP3A and CTRP3B. CTRP3 protein expression was demonstrated to be increased in the mouse OS cell lines NHOS and LM8, and it was shown to promote cell proliferation, and the effect of promoting cell proliferation is increased with the increase in CTRP3 level (30). In cartilage progenitor and endothelial cells, CTRP3 can activate the MAPK and PI3K/AKT pathways $(27,31)$, which are crucial for cell proliferation, in response to growth factors and other control signals $(32,33)$. The progression of OS is induced by a complex intracellular signaling network, and both the MAPK and PI3K/AKT pathways are key regulators of the proliferation and survival of OS cells $(34,35)$.

Exogenous CTRP3 can promote the proliferation of OS cells via the ERK1/2 pathway, and activation of the ERK1/2 pathway is associated with tumor angiogenesis. U0126, which is an inhibitor of the MAPK/ERK1/2 (MEK1/2) pathway, can block CTRP3-mediated cell proliferation (36), suggesting that CTRP3 may play an important role in the development of OS. One possible mechanism may involve the promotion of vascular endothelial cell proliferation and new blood vessel formation via the MAPK/ERK1/2 signal transduction pathway, which would accelerate tumor growth and facilitate the spread of tumor cells (Fig. 2). MEK5 also plays an important role in the occurrence and development of tumors and can be inhibited by U0126. Subsequently, MEK5 is likely to play a role in the development of OS via one of the pathways regulated by CTRP3. However, there are currently no supporting evidence. Further investigation is therefore needed to explore this possibility. A previous study demonstrated that overexpression of the micro-RNA (miR)-495-3p in human OS cell lines inhibits the proliferation, migration and invasion of OS cells by downregulating the expression of CTRP3, which is a direct target of miR-495-3p (36). CTRP3 could therefore be considered as a therapeutic target for OS. In addition, Kim et al (37) demonstrated that CTRP3 has a negative effect on osteoclast bone resorption activity both in vitro and in vivo through inhibition of osteoclast formation by acting directly on osteoclast precursors. Based on these data, the authors proposed that CTRP3 could be a potential treatment target for bone diseases associated with excessive osteoclast differentiation and bone destruction. At present, the underlying mechanism of CTRP3 effect in OS progression is unknown, and identification of the CTRP3 receptor would facilitate the investigation of its function and help determining its potential use in clinical application. These findings should be further verified in additional cell lines.

\section{The role of CTRP4 and CTRP6 in liver cancer}

Chronic infection and inflammation are primary predisposing factors for several types of tumor. For example, simultaneous 


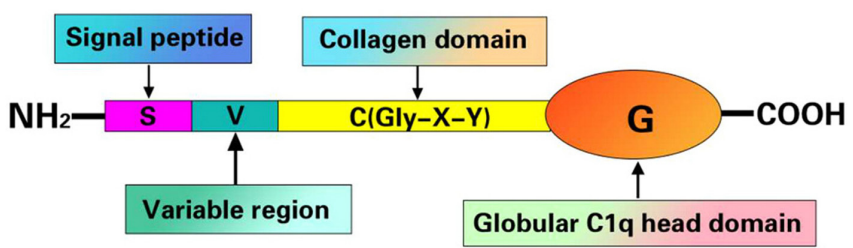

Figure 1. Structure of Clq tumor necrosis factor-related proteins [modified from Schäffler et al, 2012 (14)].

infection with hepatitis $B$ and hepatitis $C$ viruses increases the risk of developing hepatocellular carcinoma (38). In addition, inflammatory signals play key roles in tumor development and progression, such as JAK/signal transducer and activator of transcription 3 (STAT3) in multiple myeloma (39) and PPAR $\gamma /$ nuclear factor- $\kappa \mathrm{B}$ (NF- $\mathrm{kB}$ ) in lung cancer (40). CTRP4, which is highly expressed in hepatocytes and bile duct epithelial cells, is a novel tumor-promoting factor involved in the regulation of inflammatory signaling pathways in tumor cells. Minimal expression of CTRP4 mRNA has been observed in the hepatoma cell line HepG2 and the human colon cancer cell line HT29 (38). Furthermore, overexpression of CTRP4 activates the NF- $\kappa B$ signaling pathway, which in turn induces the production of multiple tumor-related cytokines, such as interleukin (IL-)6 and tumor necrosis factor- $\alpha$ (TNF- $\alpha$ ) (41).

The NF- $\mathrm{KB}$ signaling pathway induces and maintains a chronic inflammation microenvironment, which is the most important mechanism of tumorigenesis (42). The Ginkgo biloba extract EGB761 has been shown to induce apoptosis in hepatoma cells by inhibiting the NF- $\mathrm{kB} / \mathrm{p} 53$ signaling pathway (43). IL-6 is related to liver cancer and IL-6 signaling upregulates androgen receptor expression. Androgen receptors decrease the expression of tumor suppressor p53 and enhances the production of reactive oxygen species (ROS) and subsequent DNA damage and mutation, eventually contributing to malignant transformation of liver cells (44). A previous study demonstrated that inhibition of IL- 6 by estrogen can reduce the risk of liver cancer in women (45). IL-6 is also the primary cytokine that regulates the STAT3 pathway. A previous study reported that CTRP4 can activate STAT3 signaling pathway (46), which regulates the transcription of various genes, including apoptosis suppressor genes $\mathrm{Bcl}-\mathrm{xL}, \mathrm{MC1}-1$, $\mathrm{X} 2 \mathrm{AP}$ and two-way regulatory gene C-myc. Through STAT3 pathway, CTRP4 regulates the survival, proliferation, differentiation and invasion of tumor cells as well as inflammation and immunosuppression (47). A previous study in animal models have demonstrated that colorectal carcinoma cell-derived TNF- $\alpha$ can facilitate tumor growth and metastasis (48). Studies examining the effects of chemotherapy on the hepatoma cell line $\mathrm{HepG} 2$ have reported that activation of inflammatory factors by CTRP 4 can promote tumor cell survival through the activation of anti-apoptosis pathways $(14,49)$. Although there is no direct evidence that CTRP4 could induce liver cancer, it is reasonable to propose CTRP4 as a potential hepatocarcinogen that may upregulate the expression of inflammatory factors by activating the NF- $\mathrm{\kappa B} / \mathrm{STAT} 3$ signaling pathway, regulating therefore the pathological processes of liver cancer (Fig. 3).

Another member of the CTRP family, CTRP6, is also overexpressed in liver cancer. A previous study reported that

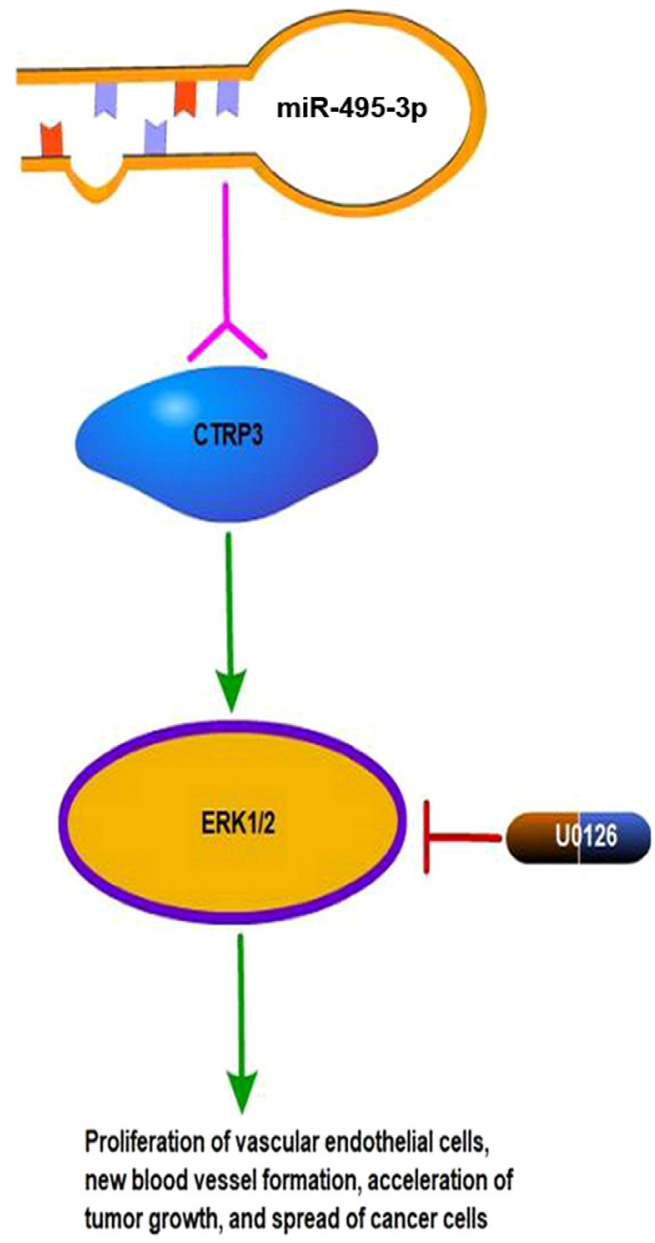

Figure 2. Schematic diagram of CTRP3-mediated promotion of osteosarcoma cell proliferation. CTRP, C1q tumor necrosis factor-related protein; miR, micro-RNA.

CTRP6 mediates a dose-dependent increase in IL-10 synthesis by macrophages following activation of ERK1/2 (24), suggesting that CTRP6 might have some anti-inflammatory effects. In addition, CTRP6 inhibits the IL-8/VEGF pathway to inhibit the proliferation and metastasis of ovarian cancer cells (50). However, in Hep3B hepatoma cells, CTRP6 knockout triggers apoptosis and inhibits cell migration and invasion (51), suggesting that CTRP6 could have either carcinogenic or anti-tumor effects depending on the type of cancer. Overexpression of CTRP6 in hepatoma cells not only facilitates the phosphorylation of AKT in sinusoidal endothelial cells but also promotes tumor angiogenesis and inhibits the apoptosis of hepatocellular carcinoma cells (52). Furthermore, inhibition of CTRP6 blocks the migration and survival of hepatocellular carcinoma cells by inactivating the AKT signaling pathway (49). In xenotransplants of hepatoma cells, CTRP6 reduces the area of central intravascular necrosis by accelerating tumor neovascularization (53). In a study on clear cell renal cell carcinoma (ccRCC), Lin et al demonstrated that CTRP6 mediates tumorigenesis by modulating the cell cycle at the G2M checkpoint, epithelial-mesenchymal transition and angiogenesis. Furthermore, CTRP6 may also participate in tumor progression by activating the AKT and ERK1/2 signaling pathways (54). According to these findings, the underlying mechanism of CTRP6 in hepatoma cells is likely 


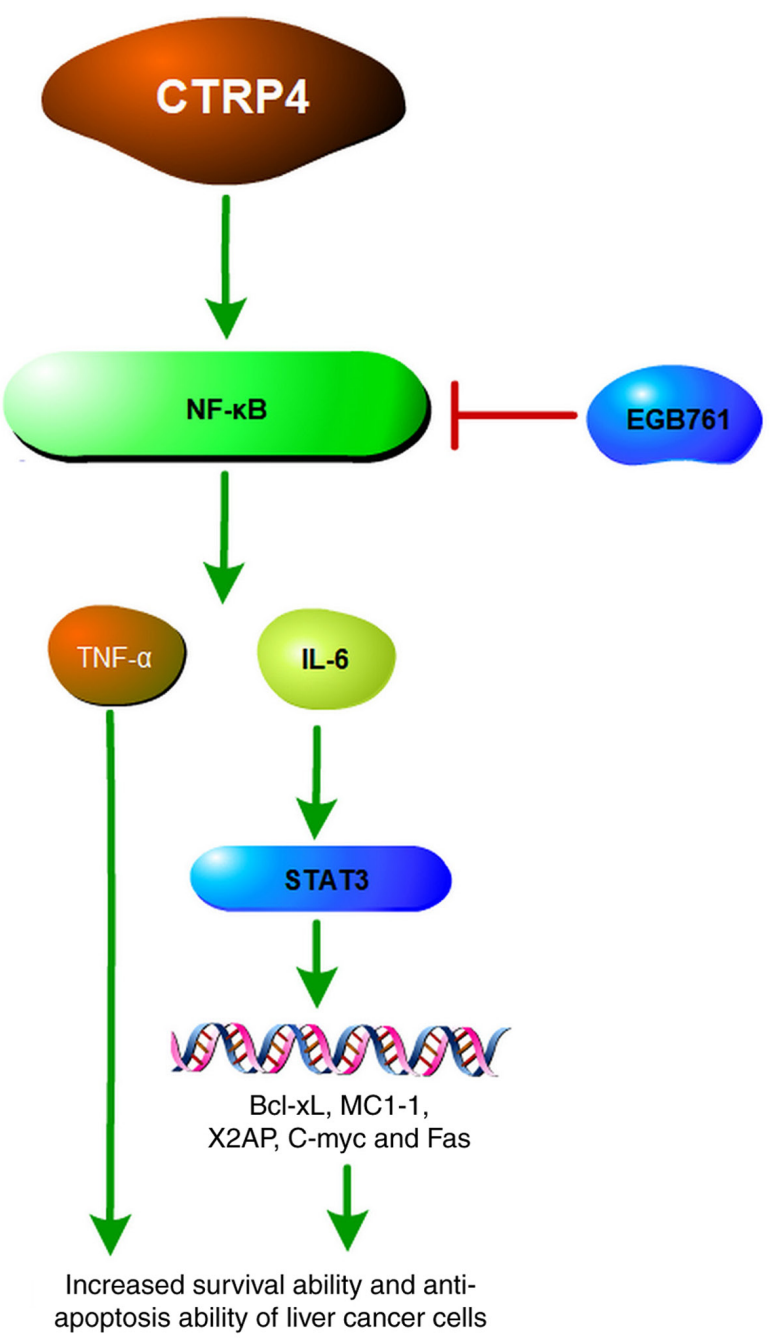

Figure 3. Schematic diagram of CTRP4-mediated induction of the development and progression of liver cancer. CTRP, C1q tumor necrosis factor-related protein; NF- $\kappa \mathrm{B}$, nuclear factor- $\kappa \mathrm{B}$; TNF- $\alpha$, tumor necrosis factor- $\alpha$; IL-6, interleukin-6; EGB761, Ginkgo biloba extract; STAT3, signal transducer and activator of transcription 3 .

to be similar to that in ccRCC; however, further investigation is required. Modulating the levels of secretory CTRP6 may therefore be considered as a new treatment approach for liver cancer.

\section{Roles of CTRP4 and CTRP6 in colon cancer}

Colon cancer is a primary malignant tumor of the digestive tract. The risk factors for colon cancer are complex and diverse, and the mechanisms underlying its development and progression remain unclear. However, it is well known that some inflammatory factors are implicated in the development of colon cancer, and a variety of inflammatory mediators, including IL-6, TNF- $\alpha$ and IL-10, have been reported to be involved in regulating the progression and metastasis of colon cancer $(55,56)$. Previous studies have reported an increased expression of CTRP6 in colon cancer tissues, regardless of patient sex and age, tumor size, degree of differentiation and depth of invasion (57). In addition, CTRP6 overexpression was demonstrated to decrease the expression levels of pro-inflammatory factors, such as IL- $1 \beta$, IL- 6 and TNF- $\alpha$, and increase the expression of the anti-inflammatory factor IL-10 (58). A decrease in CTRP6 expression increases the aggressiveness of ovarian cancer cells, while CTRP6 overexpression inhibits the proliferation of cancer cells (51). Conversely, CTRP6 knockout in glomerular mesangial cells inhibits the generation of intracellular ROS and inflammation (59). The expression level of CTRP6 is significantly increased in high glucose-induced glomerular mesangial cells, and CTRP6 knockdown causes significant decrease in TNF- $\alpha$, IL- $1 \beta$ and IL- 6 production levels in these cells. Treatment with LY294002, an inhibitor of Akt, reverses the induction effects of CTRP6 overexpression on ROS production, inflammation and extracellular matrix accumulation in mesangial cells. In addition, activation of the $\mathrm{AKT} / \mathrm{NF}-\kappa \mathrm{B}$ pathway promotes the growth, migration and invasion of colon cancer cells (60). Because of these discrepancies, the specific underlying mechanisms of CTRP6 in colon cancer remain to be determined. However, CTRP6 is closely related to the occurrence and development of colon cancer.

CTRP4 expression is increased in the human colon cancer cell line HT29 where it functions as a dual activator of NF- $\mathrm{\kappa B}$ and IL-6 (41), which are important regulators of cancer development and progression. However, a previous study on mice has shown that overexpression of CTRP4 not only inhibits the production of IL- 6 but also inhibits the initiation of colon cancer induced by DSS/AOM, which is a method used to extablish colon cancer model through drugs (47). These findings were further supported by in vitro experiments. For example, the human recombinant CTRP4 can inhibit the lipopolysaccharide-induced overexpression of the inflammatory factor TNF- $\alpha$ in macrophages, suggesting that CTRP4 may have a potential protective factor in inflammatory bowel disease (61), which is an independent risk factor for colon cancer (62). The role of CTRP4 in colon cancer remains therefore controversial. Although the therapeutic significance of CTRP4 in colon cancer has yet to be determined, it is clear that CTRP4 is involved in the regulation of the inflammatory network and that it serves a crucial role in the development of colon cancer.

\section{GBM and CTRP8}

GBM is a malignant brain cancer with the highest malignancy grade among astrocytic tumors (63). CTRP8 was recently discovered as a novel ligand of the $\mathrm{G}$ protein-coupled receptor relaxin family peptide receptor-1 (RXFP1) and was detected in fatal GBM cases (64). CTRP8 can activate the PI3K, PKC and JAK2/STAT3 signaling cascade in brain tumor cells (65) Owing to its important molecular role in cancer, RXFP1 has been extensively investigated in breast, endometrial, prostate and thyroid cancers. The effects of RXFP1 activation in GBM cells include increased cell motility, tumor expansion, tissue invasion and metastasis (66), tumor growth and angiogenesis (67). The mechanism underlying CTRP8/RXFP1-mediated aggressiveness of GBM tumors involves the RXFP1/PI3K/PKC/cathepsin B signaling cascade. In GBM cells, two biologically active peptides with homologous sequences in the $\mathrm{N}$-terminal region of the $\mathrm{Clq}$ globular domain of CTRP8 activate RXFP1 via cAMP and PI3K-PKC/PKC-ERK1/2 signaling. In addition, activation of RXFP1 by CTRP8 increases the production and secretion of 
cathepsin-B protein and increases the invasiveness of GBM cells via the laminin matrix (68). The U251 GMB cell line and HEK293 cells, which do not express RXFP1 but have exogenous expression of RXFP2, do not respond to elevated cAMP, indicating that the RXFP1-mediated signal activation is cell-type specific (27). In addition, the inhibition of cathepsin B secretion and invasion by CTRP6 suggests that CTRP6 may act as a competitor of CTRP8 for binding to RXFP1 and may subsequently block CTRP8 signaling as the concentration of CTRP6 increases (66). We therefore hypothesized that CTRP6 may affect the development and progression of GBM. However, further clinical verification is required. A recent study by Thanasupawat et al (64) reported a novel role for CTRP8 in the protection of GBM cells from DNA alkylation damage induced by the chemotherapeutic drug temozolomide (TMZ) (69). This study showed that the mechanism underlying CTRP8-induced chemotherapy resistance involves activation of the newly discovered RXFP1/STAT3 signaling pathway and upregulation of a new target of this pathway, $\mathrm{N}$-methylpurine DNA glycosylase. This mechanism boosts the DNA repair system and consequently promotes activation of the anti-apoptotic pathway involving Bcl-2 and Bcl-XL in GBM cells. Treatment with TMZ increases cell resistance to DNA alkylation via CTRP8/-RXFP1/-STAT3, thereby improving the survival rates of patients with GBM. The effects of CTRP8 in GBM are summarized in Fig. 4.

\section{Tumor-related roles of the other CTRP family members}

A recent study demonstrated that CTRP1 mRNA expression is significantly higher in GBM tissues compared with normal tissues. Knockout of the CTRPI gene significantly inhibits the proliferation and migration of human GBM cells, suggesting that CTRP1 may promote the progression of human GBM, thereby leading to a poor prognosis in patients (70). Regarding the development and progression of chondroblastoma, CTRP3 induces the activation of liver AKT signaling pathway and inhibits the expression of liver gluconeogenesis enzymes, thus reducing liver gluconeogenesis and lowering glucose levels, which promote the proliferation of chondrogenic cells (27,71). Qu et al (72) reported CTRP8 overexpression in gastric cancer as well as its involvement in the proliferation and migration of gastric cancer cells. CTRP6 downregulation induces cell cycle arrest at the G2M checkpoint and apoptosis in gastric cancer cells. This finding indicates that CTRP6 increases the sensitivity of gastric cancer cells to apoptosis. In addition, as a direct target of miR-29b, CTRP6 regulates tumor progression and may function as either a tumor promoter or a tumor suppressor, depending on the type of cell or tissue. Downregulation of CTRP6 in MCF-7 breast cancer cells increases tumor aggressiveness (73), while CTRP6 overexpression in SKOV3, 3AO and HO8910 epithelial ovarian cancer cells inhibits proliferation and migration by blocking the IL-8/VEGF pathway. Furthermore, a previous study reported that CTRP6 expression is increased in ccRCC and is positively correlated with disease stage (51). A very recent study demonstrated that inhibition of CTRP6 can attenuate cell proliferation, migration, invasion and promote apoptosis in vitro and in vivo in non-small cell lung cancer (74). However, in a previous

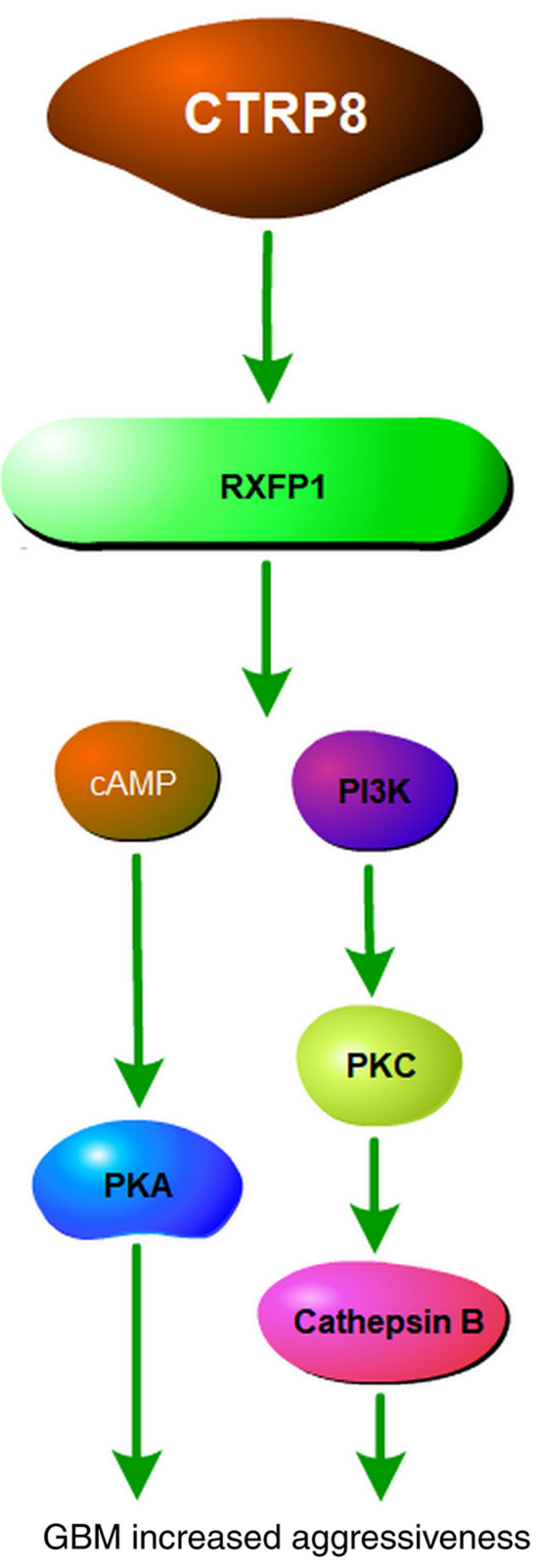

Figure 4. Schematic diagram of the role CTRP8 in GBM.GBM, glioblastoma; CTRP, C1q tumor necrosis factor-related protein; RXFP1, relaxin family peptide receptor- 1 .

study, aberrant C1QTNF6 expression is implicated in terrible prognosis accompanied with damage of cell potential in lung adenocarcinoma (75). CRTP6 seems therefore to play opposite roles in different studies. All these studies are still at early stages and the underlying mechanisms of numerous CTRPs remain unknown.

\section{Conclusions}

By activating numerous signaling pathways, the CTRP family plays important roles in human cancers in various tissues and organs, such as the digestive tract, brain and bone. CTRP3 promotes angiogenesis in bone tumors by activating the ERK1/2 pathway, thereby accelerating tumor growth and promoting the spread of cancer cells (27). CTRP4 modulates tumor development and progression by activating the $\mathrm{NF}-\kappa \mathrm{B} / \mathrm{STAT} 3$ signaling 


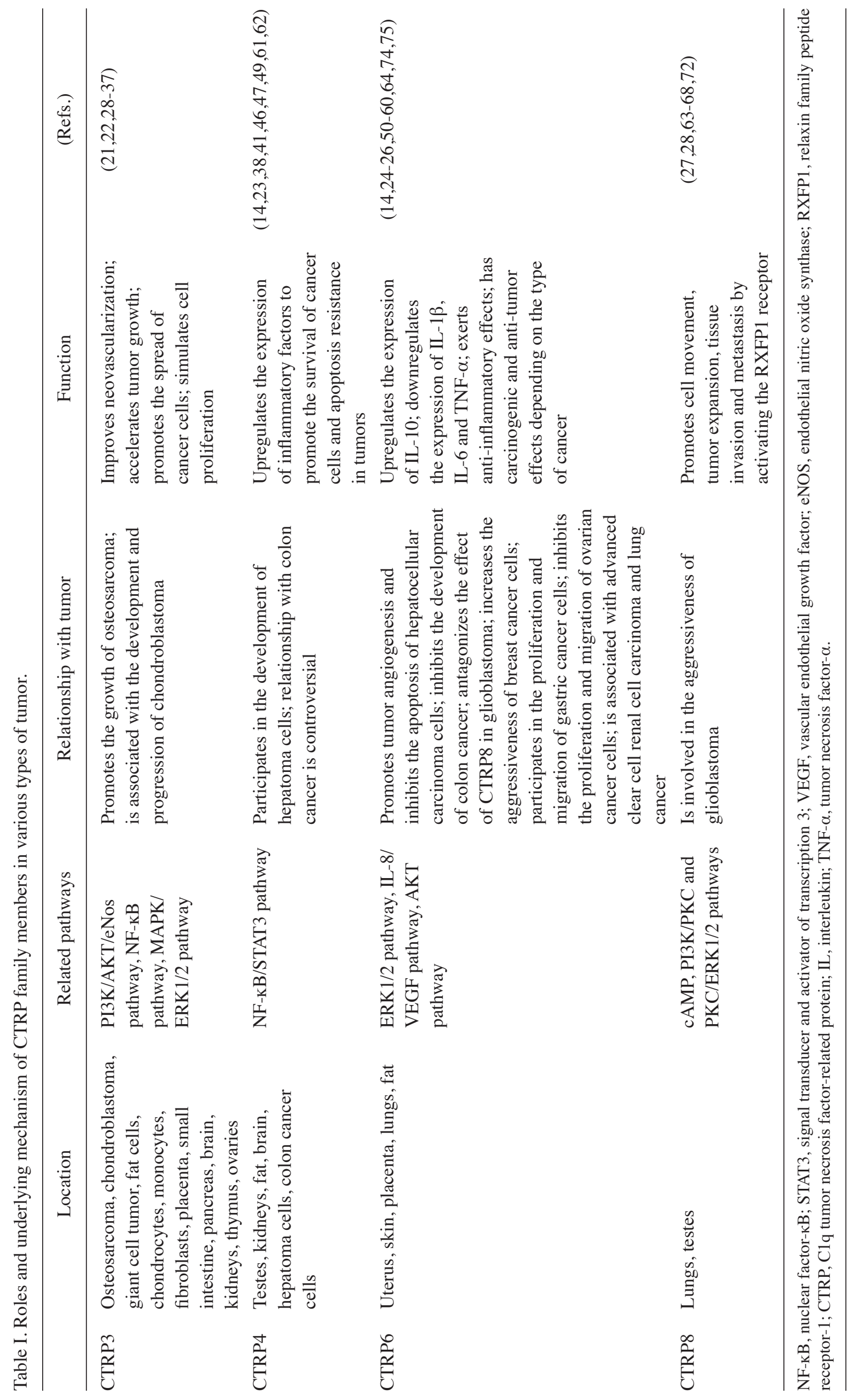


pathway in hepatocytes and colon cancer cells and inducing the expression of multiple tumor-associated cytokines (10). CTRP6 exhibits both carcinogenic and anti-tumor effects, which are cancer-type dependent. CTRP6 is expressed in colon cancer and ovarian cancer cells where it exerts different functions. In colon cancer, CTRP6 inhibits tumor development and progression by decreasing pro-inflammatory factors and increasing anti-inflammatory factors (26). In ovarian cancer, CTRP6 inhibits the proliferation and migration of cancer cells by blocking the expression of IL-8 and vascular endothelial growth factor pathways (52). Conversely, overexpression of CTRP6 in hepatocellular carcinoma increases tumor angiogenesis, whereas CTRP6 silencing can activate the AKT signaling pathway. Subsequently, CTRP6 promotes apoptosis in hepatoma cells and prevents cell invasion. The effects of CTRP8 have been reported in brain tumors. CTRP8 binds to RXFP1 to activate the $\mathrm{RXFP} 1 / \mathrm{PI} 3 \mathrm{~K} / \mathrm{PKC} /$ cathepsin B signaling pathway and consequently increases the invasiveness of GBM cells (29). The roles of these CTRP family members have also been investigated in other cancers, such as gastric cancer, ovarian cancer and renal cell carcinoma, and are summarized in Table I. CTRP family members are widely expressed, which provides the basis for their function in multi-system tumors. However, studies examining CTRPs are still insufficient, and further investigation is crucial to expand our understanding on CTRP biological functions and CTRP-related diseases. At present, studies demonstrated that CTRPs have the potential to be considered as therapeutic targets in numerous types of cancer, which offers glimmer of hope for potential treatment strategies.

\section{Acknowledgements}

Not applicable.

\section{Funding}

This study was supported by the Hebei Province Talent Training Project (grant no. A201802018).

\section{Availability of data and materials}

Not applicable.

\section{Authors' contributions}

MK wrote the manuscript. YG, XG, YX and YY reviewed and revised the manuscript. All authors have read and approved the final manuscript.

\section{Ethical approval and consent to participate}

Not applicable.

\section{Patient consent for publication}

Not applicable.

\section{Competing interests}

The authors declare that they have no competing interests.

\section{References}

1. Archambault AN, Su YR, Jeon J, Thomas M, Lin Y, Conti DV, Win AK, Sakoda LC, Lansdorp-Vogelaar I, Peterse EFP, et al: Cumulative burden of colorectal cancer-associated genetic variants is more strongly associated with early-onset vs. late-onset cancer. Gastroenterology 158: 1274-1286.e12, 2020.

2. Sagnelli E, Macera M, Russo A, Coppola N and Sagnelli C: Epidemiological and etiological variations in hepatocellular carcinoma. Infection 48: 7-17, 2020.

3. Işçi Bostanci E, Durmuş Y, Duru Çöteli SA, Kayikçioğlu F and Boran N: Outcomes of the conservative management of the patients with endometrial intraepithelial neoplasia/endometrial cancer: Wait or treat!. Turk J Med Sci: May 20,2021 (Epub ahead of print).

4. Heyn GS, Corrêa LH and Magalhães KG: The impact of adipose tissue-derived miRNAs in metabolic syndrome, obesity, and cancer. Front Endocrinol (Lausanne) 11: 563816, 2020.

5. Marino N, German R, Rao X, Simpson E, Liu S, Wan J, Liu Y, Sandusky G, Jacobsen M, Stoval M, et al: Upregulation of lipid metabolism genes in the breast prior to cancer diagnosis. NPJ Breast Cancer 6: 50, 2020.

6. Philp LK, Rockstroh A, Lehman M, Sadowski MC, Bartonicek N, Wade JD, Otvos L and Nelson CC: Adiponectin receptor activation inhibits prostate cancer xenograft growth. Endocr Relat Cancer 27: 711-729, 2020

7. Llanos AAM, Yao S, Singh A, Aremu JB, Khiabanian H, Lin Y, Omene C, Omilian AR, Khoury T, Hong CC, et al: Gene expression of adipokines and adipokine receptors in the tumor microenvironment: Associations of lower expression with more aggressive breast tumor features. Breast Cancer Res Treat 185: 785-798, 2021.

8. Wu X, Long X, Yang C, Chen H, Sharkey C, Rashid K, Hu M, Liu Y, Huang Q, Chen Q, et al: Icaritin reduces prostate cancer progression via inhibiting high-fat diet-induced serum adipokine in TRAMP mice model. J Cancer 11: 6556-6564, 2020.

9. Wong GW, Krawczyk SA, Kitidis-Mitrokostas C, Revett T, Gimeno R and Lodish HF: Molecular, biochemical and functional characterizations of $\mathrm{C} 1 \mathrm{q} / \mathrm{TNF}$ family members: Adipose-tissue-selective expression patterns, regulation by PPAR-gamma agonist, cysteine-mediated oligomerizations, combinatorial associations and metabolic functions. Biochem J 416: 161-177, 2008.

10. Sarver DC, Stewart AN, Rodriguez S, Little HC, Aja S and Wong GW: Loss of CTRP4 alters adiposity and food intake behaviors in obese mice. Am J Physiol Endocrinol Metab 319: E1084-E1100, 2020.

11. Shanaki M, Shabani P, Goudarzi A, Omidifar A, Bashash D and Emamgholipour S: The Clq/TNF-related proteins (CTRPs) in pathogenesis of obesity-related metabolic disorders: Focus on type 2 diabetes and cardiovascular diseases. Life Sci 256: $117913,2020$.

12. Rasooli Tehrani A, Gholipour S, Sharifi R, Yadegari S, Abbasi-Kolli M and Masoudian N: Plasma levels of CTRP-3, CTRP-9 and apelin in women with multiple sclerosis. J Neuroimmunol 333: 576968, 2019.

13. Kishore U, Gaboriaud C, Waters P, Shrive AK, Greenhough TJ, Reid KB, Sim RB and Arlaud GJ: Clq and tumor necrosis factor superfamily: Modularity and versatility. Trends Immunol 25: 551-561, 2004.

14. Schäffler A and Buechler C: CTRP family: Linking immunity to metabolism. Trends Endocrinol Metab 23: 194-204, 2012

15. Wong GW, Wang J, Hug C, Tsao TS and Lodish HF: A family of Acrp30/adiponectin structural and functional paralogs. Proc Natl Acad Sci USA 101: 10302-10307, 2004

16. Tan SY, Little HC, Sarver DC, Watkins PA and Wong GW: CTRP12 inhibits triglyceride synthesis and export in hepatocytes by suppressing HNF-4 $\alpha$ and DGAT2 expression. FEBS Lett 594: 3227-3239, 2020

17. Lei $\mathrm{X}$ and Wong GW: Clq/TNF-related protein 2 (CTRP2) deletion promotes adipose tissue lipolysis and hepatic triglyceride secretion. J Biol Chem 294: 15638-15649, 2019.

18. Moradi N, Najafi M, Sharma T, Fallah S, Koushki M, Peterson JM, Meyre D and Fadaei R: Circulating levels of CTRP3 in patients with type 2 diabetes mellitus compared to controls: A systematic review and meta-analysis. Diabetes Res Clin Pract 169: 108453, 2020.

19. Qian M, Yang Q, Li J, Zhao B, Zhang Y and Zhao Y: C1q/TNF-related protein-9 alleviates airway inflammation in asthma. Int Immunopharmacol 81: 106238, 2020. 
20. Peterson JM, Wei Z and Wong GW: CTRP8 and CTRP9B are novel proteins that hetero-oligomerize with C1q/TNF family members. Biochem Biophys Res Commun 388: 360-365, 2009.

21. Schäffler A, Ehling A, Neumann E, Herfarth H, Paul G, Tarner I, Gay S, Schölmerich J and Müller-Ladner U: Genomic organization, promoter, amino acid sequence, chromosomal localization, and expression of the human gene for CORS-26 (collagenous repeat-containing sequence of $26-\mathrm{kDa}$ protein). Biochim Biophys Acta 1630: 123-129, 2003.

22. Maeda T, Abe M, Kurisu K, Jikko A and Furukawa S: Molecular cloning and characterization of a novel gene, CORS26, encoding a putative secretory protein and its possible involvement in skeletal development. J Biol Chem 276: 3628-3634, 2001

23. Byerly MS, Petersen PS, Ramamurthy S, Seldin MM, Lei X, Provost E, Wei Z, Ronnett GV and Wong GW: Clq/TNF-related protein 4 (CTRP4) is a unique secreted protein with two tandem Clq domains that functions in the hypothalamus to modulate food intake and body weight. J Biol Chem 289: 4055-4069, 2014

24. Li Q, Wu J, Xi W, Chen X, Wang W, Zhang T, Yang A and Wang T: Ctrp4, a new adipokine, promotes the differentiation of osteoblasts. Biochem Biophys Res Commun 512: 224-229, 2019.

25. Kirketerp-Møller N, Bayarri-Olmos R, Krogfelt KA and Garred P: C1q/TNF-related protein 6 is a pattern recognition molecule that recruits collectin-11 from the complement system to ligands. J Immunol 204: 1598-1606, 2020.

26. Lei X, Seldin MM, Little HC, Choy N, Klonisch T and Wong GW: C1q/TNF-related protein 6 (CTRP6) links obesity to adipose tissue inflammation and insulin resistance. J Biol Chem 292 14836-14850, 2017.

27. Akiyama H,Furukawa S, Wakisaka $S$ and Maeda T: CTRP3/cartducin promotes proliferation and migration of endothelial cells. Mol Cell Biochem 304: 243-248, 2007.

28. Zhao G, Zhang L, Qian D, Sun Y and Liu W: miR-495-3p inhibits the cell proliferation, invasion and migration of osteosarcoma by targeting Clq/TNF-related protein 3 . Onco Targets Ther 12: 6133-6143, 2019.

29. Glogowska A, Thanasupawat T, Beiko J, Pitz M, HombachKlonisch S and Klonisch T: Novel CTRP8-RXFP1-JAK3-STAT3 axis promotes $\mathrm{Cdc} 42$-dependent actin remodeling for enhanced filopodia formation and motility in human glioblastoma cells. Mol Oncol, May 7, 2021 (Epub ahead of print).

30. Akiyama H, Furukawa $S$, Wakisaka $S$, Maeda $T$ and Maeda T: Elevated expression of CTRP3/cartducin contributes to promotion of osteosarcoma cell proliferation. Oncol Rep 21: 1477-1481, 2009

31. Akiyama H, Furukawa S, Wakisaka S and Maeda T: Cartducin stimulates mesenchymal chondroprogenitor cell proliferation through both extracellular signal-regulated kinase and phosphatidylinositol 3-kinase/Akt pathways. FEBS J 273: 2257-2263, 2006.

32. Li JM, Zhang X, Nelson PR, Odgren PR, Nelson JD, Vasiliu C, Park J, Morris M, Lian J, Cutler BS and Newburger PE: Temporal evolution of gene expression in rat carotid artery following balloon angioplasty. J Cell Biochem 101: 399-410, 2007.

33. Hill CS and Treisman R: Transcriptional regulation by extracellular signals: Mechanisms and specificity. Cell 80: 199-211, 1995.

34. Seger R and Krebs EG: The MAPK signaling cascade. FASEB J 9: 726-735, 1995

35. Coltella N, Manara MC, Cerisano V, Trusolino L, Di Renzo MF, Scotlandi K and Ferracini R: Role of the MET/HGF receptor in proliferation and invasive behavior of osteosarcoma. FASEB J 17: 1162-1164, 2003.

36. Bentov I, LeRoith D and Werner H: The WT1 Wilms' tumor suppressor gene: A novel target for insulin-like growth factor-I action. Endocrinology 144: 4276-4279, 2003.

37. Kim JY, Min JY, Baek JM, Ahn SJ, Jun HY, Yoon KH, Choi MK, Lee MS and Oh J: CTRP3 acts as a negative regulator of osteoclastogenesis through AMPK-c-Fos-NFATc1 signaling in vitro and RANKL-induced calvarial bone destruction in vivo. Bone 79: 242-251, 2015

38. Fattovich G, Stroffolini T, Zagni I and Donato F: Hepatocellular carcinoma in cirrhosis: Incidence and risk factors. Gastroenterology 127 (5 Suppl 1): S35-S50, 2004.

39. Jung YY, Um JY, Nasif O, Alharbi SA, Sethi G and Ahn KS Blockage of the JAK/STAT3 signaling pathway in multiple myeloma by leelamine. Phytomedicine: Apr 15, 2021 (Epub ahead of print).

40. Hang T, Yang L, Zhang X, Li J, Long F, Zhu N, Li Y, Xia J, Zhang Y, Zhang $\mathrm{P}$, et al: Peroxisome proliferator-activated receptor $\gamma$ improves pemetrexed therapeutic efficacy in non-squamous non-small cell lung cancer. Am J Transl Res 13: 2296-2307, 2021.
41. Li Q, Wang L, Tan W, Peng Z, Luo Y, Zhang Y, Zhang G, Na D, Jin P, Shi T, et al: Identification of ClqTNF-related protein 4 as a potential cytokine that stimulates the STAT3 and NF- $\mathrm{BB}$ pathways and promotes cell survival in human cancer cells. Cancer Lett 308: 203-214, 2011.

42. Grivennikov S, Karin E, Terzic J, Mucida D, Yu GY, Vallabhapurapu S, Scheller J, Rose-John S, Cheroutre H, Eckmann L and Karin M: IL-6 and Stat3 are required for survival of intestinal epithelial cells and development of colitis-associated cancer. Cancer Cell 15: 103-113, 2009.

43. Xia Y, Shen S and Verma IM: NF-kappaB, an active player in human cancers. Cancer Immunol Res 2: 823-830, 2014.

44. Wang R, Shao X, Yang J,Liu Z, Chew L and Shao Y: Ginkgo biloba extract mechanism inhibits hepatocellular carcinoma through the nuclear factor- $\kappa \mathrm{B} / \mathrm{p} 53$ signaling pathway. J Environ Pathol Toxicol Oncol 39: 179-189, 2020.

45. Yang YM, Kim SY and Seki E: Inflammation and liver cancer: Molecular mechanisms and therapeutic targets. Semin Liver Dis 39: 26-42, 2019.

46. Naugler WE, Sakurai T,Kim S, Maeda S, Kim K, Elsharkawy AM and Karin M: Gender disparity in liver cancer due to sex differences in MyD88-dependent IL-6 production. Science 317: 121-124, 2007.

47. Li Y, Ye L, Jia G, Chen H, Yu L and Wu D: C1q/TNF-related protein 4 induces signal transducer and activator of transcription 3 pathway and modulates food intake. Neuroscience 429: 1-9, 2020.

48. Yu H, Lee H, Herrmann A, Buettner R and Jove R: Revisiting STAT3 signalling in cancer: New and unexpected biological functions. Nat Rev Cancer 14: 736-746, 2014.

49. Balkwill F: TNF-alpha in promotion and progression of cancer. Cancer Metastasis Rev 25: 409-416, 2006.

50. Kim MJ, Lee W, Park EJ and Park SY: C1qTNF-related protein-6 increases the expression of interleukin-10 in macrophages. Mol Cells 30: 59-64, 2010.

51. Wang L, Liu Z, Duan L, Ma B and Sun Z: Clq tumor necrosis factor-related protein 6 (CTRP6) inhibits the proliferation and migration of ovarian cancer cells. Xi Bao Yu Fen Zi Mian Yi Xue Za Zhi 31: 1664-1668, 2015 (In Chinese).

52. Wan X, Zheng C and Dong L: Inhibition of CTRP6 prevented survival and migration in hepatocellular carcinoma through inactivating the AKT signaling pathway. J Cell Biochem 120: 17059-17066, 2019.

53. Takeuchi T, Adachi $\mathrm{Y}$ and Nagayama T: Expression of a secretory protein CTRP6, a C1qTNF family member, in hepatocellular carcinoma. Anal Cell Pathol (Amst) 34: 113-121, 2011

54. Lin W, Chen X, Chen T, Liu J, Ye Y, Chen L, Qiu X, Chia-Hsien Cheng J, Zhang L, Wu J and Qiu S: C1QTNF6 as a novel diagnostic and prognostic biomarker for clear cell renal cell carcinoma. DNA Cell Biol 39: 1000-1011, 2020.

55. Milone M, Desiderio A, Velotti N, Manigrasso M, Vertaldi S, Bracale U, D'Ambra M, Servillo G, De Simone G, De Palma FDE, et al: Surgical stress and metabolic response after totally laparoscopic right colectomy. Sci Rep 11: 9652, 2021.

56. James S, Aparna JS,Babu A,Paul AM,Lankadasari MB,AthiraSR, Kumar SS, Vijayan Y, Namitha NN, Mohammed S, et al: Cardamonin attenuates experimental colitis and associated colorectal cancer. Biomolecules 11: 661, 2021.

57. Gou JX, Jiang XJ, Qu HX and Cui YX: Expression of Complement Clq/Tumor Necrosis Factor Related Protein 6 in Colon Cancer. J Gastroenterol Hepatol 28: 313-316, 2019.

58. Lei H, Wu D, Wang JY, Li L, Zhang CL, Feng H, Fu FY and Wu LL: C1q/tumor necrosis factor-related protein-6 attenuates post-infarct cardiac fibrosis by targeting RhoA/MRTF-A pathway and inhibiting myofibroblast differentiation. Basic Res Cardiol 110: 35, 2015

59. Xu E, Yin C, Yi X and Liu Y: Knockdown of CTRP6 inhibits high glucose-induced oxidative stress, inflammation and extracellular matrix accumulation in mesangial cells through regulating the Akt/NF-кB pathway. Clin Exp Pharmacol Physiol 47: 1203-1211, 2020.

60. Wang YP, Zhao YJ and Kong XL: A metalloproteinase of the disintegrin and metalloproteinases and the ThromboSpondin Motifs 6 as a novel marker for colon cancer: Functional experiments. Genet Mol Biol 43: e20190266, 2020.

61. Cao L, Tan W, Chen W, Huang H, He M, Li Q, Zhu X and Wang L: CTRP4 acts as an anti-inflammatory factor in macrophages and protects against endotoxic shock. Eur J Immunol 51: 380-392, 2021.

62. Gillen CD, Walmsley RS, Prior P, Andrews HA and Allan RN: Ulcerative colitis and Crohn's disease: A comparison of the colorectal cancer risk in extensive colitis. Gut 35: 1590-1592, 1994 
63. Lv W, Li Q, Jia B, He Y, Ru Y, Guo Q, Li X and Lin W: Differentiated embryonic chondrocyte-expressed gene 1 promotes temozolomide resistance by modulating the SP1-MGMT ax is in glioblastoma. Am J Transl Res 13: 2331-2349, 2021.

64. Thanasupawat T, Glogowska A, Burg M, Wong GW, Hoang-Vu C, Hombach-Klonisch S and Klonisch T: RXFP1 is targeted by complement $\mathrm{Clq}$ tumor necrosis factor-related factor 8 in brain cancer. Front Endocrinol (Lausanne) 6: 127, 2015.

65. Guo X, Liu Y, Huang X, Wang Y, Qu J and Lv Y: Serum relaxin as a diagnostic and prognostic marker in patients with epithelial ovarian cancer. Cancer Biomark 21: 81-87, 2017.

66. Klonisch T, Bialek J, Radestock Y, Hoang-Vu C and Hombach-Klonisch S: Relaxin-like ligand-receptor systems are autocrine/paracrine effectors in tumor cells and modulate cancer progression and tissue invasiveness. Adv Exp Med Biol 612: 104-118, 2007.

67. Silvertown JD, Symes JC, Neschadim A, Nonaka T, Kao JC, Summerlee AJ and Medin JA: Analog of $\mathrm{H} 2$ relaxin exhibits antagonistic properties and impairs prostate tumor growth. FASEB J 21: 754-65, 2007.

68. Klonisch T, Glogowska A, Thanasupawat T, Burg M, Krcek J, Pitz M, Jaggupilli A, Chelikani P, Wong GW and HombachKlonisch S: Structural commonality of C1q TNF-related proteins and their potential to activate relaxin/insulin-like family peptide receptor 1 signalling pathways in cancer cells. $\mathrm{Br}$ J Pharmacol 174: 1025-1033, 2017.

69. Thanasupawat T, Glogowska A, Burg M, Krcek J, Beiko J, Pitz M, Zhang GJ, Hombach-Klonisch S and Klonisch T: Clq/TNF-related peptide 8 (CTRP8) promotes temozolomide resistance in human glioblastoma. Mol Oncol 12: 1464-1479, 2018.
70. Chen L and Su G: Identification of CTRP1 as a prognostic biomarker and oncogene in human glioblastoma. Biomed Res Int 2019: 2582416, 2019.

71. Maeda T, Jikko A, Abe M, Yokohama-Tamaki T, Akiyama H, Furukawa S, Takigawa M and Wakisaka S: Cartducin, a paralog of Acrp30/adiponectin, is induced during chondrogenic differentiation and promotes proliferation of chondrogenic precursors and chondrocytes. J Cell Physiol 206: 537-544, 2006.

72. Qu HX, Cui L, Meng XY, Wang ZJ, Cui YX, Yu YP, Wang D and Jiang XJ: C1QTNF6 is overexpressed in gastric carcinoma and contributes to the proliferation and migration of gastric carcinoma cells. Int J Mol Med 43: 621-629, 2019.

73. Wang C, Gao C, Zhuang JL, Ding C and Wang Y: A combined approach identifies three mRNAs that are down-regulated by micro RNA-29b and promote invasion ability in the breast cancer cell line MCF-7. J Cancer Res Clin Oncol 138: $2127-$ 2136, 2012.

74. Zhang W and Feng G: C1QTNF6 regulates cell proliferation and apoptosis of NSCLC in vitro and in vivo. Biosci Rep 41: BSR20201541, 2021.

75. Han M, Wang B, Zhu M and Zhang Y: C1QTNF6 as a novel biomarker regulates cellular behaviors in A549 cells and exacerbates the outcome of lung adenocarcinoma patients. In Vitro Cell Dev Biol Anim 55: 614-621, 2019. 